Pathologe 2010 · [Suppl 2] 31:132-133

DOI 10.1007/s00292-010-1343-9

Online publiziert: 15. August 2010

(c) Springer-Verlag 2010

E.C. Obermann · N. Müller · A. Rufle · T. Menter · S. Dirnhofer · A. Tzankov

Institut für Pathologie, Universitätsspital Basel, Schweiz

\title{
Klonale Verwandtschaft von Hodgkin-Lymphomen und deren Rezidiven
}

der auftretenden HL um ein Rezidiv des ersten HL oder um ein zweites De-novoHL handelt.

\section{Material und Methoden}

Wir schlossen 11 Patienten mit z. T. mehreren Rezidiven in unsere Studie ein. Die Rezidive wurden in Frührezidive (inner- halb von 12 Monaten nach dem vorhergehenden Lymphom) und Spätrezidive (später als 12 Monate nach dem vorhergehenden Lymphom) unterteilt. Die Hodgkin- und Reed-Sternberg-Zellen wurden nach immunhistochemischer Färbung für CD3o lasergestützt mikrodisseziert (PALM MicroBeam-System, Zeiss, Deutschland). Die Länge der Schwerkettenimmunwandt sind $d . h$ ob es sich bei dem wie-

Patient

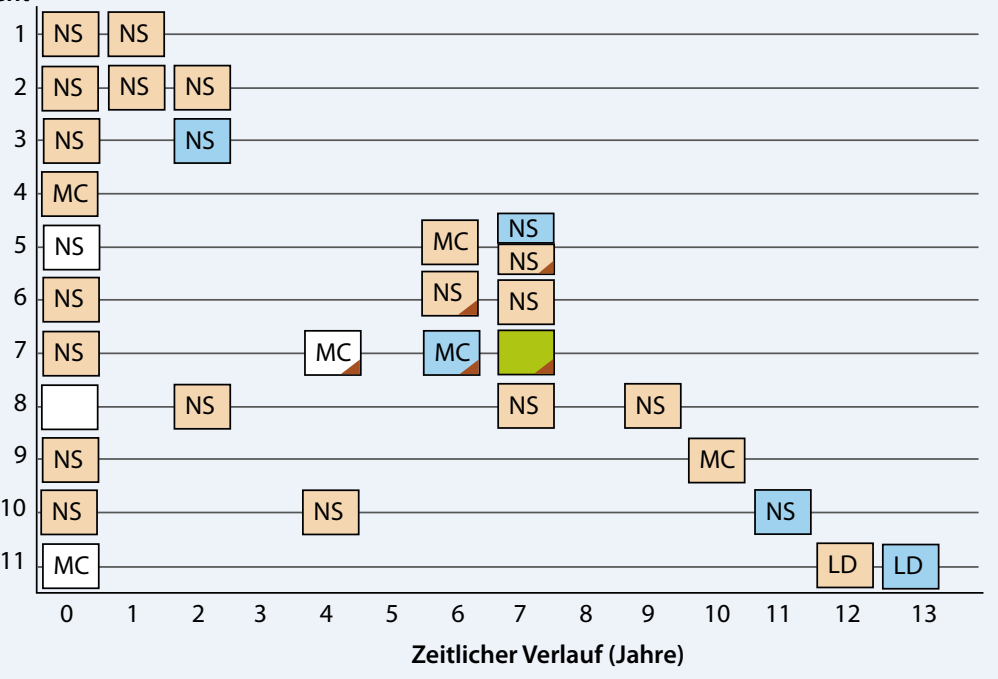

FR3 IgH Fragmentlängen

112 bp

$145 \mathrm{bp}$

$110 \rightarrow 125 \mathrm{bp}$

$126 \mathrm{bp}$

$134 \rightarrow 107 \mathrm{bp}$

$94 \mathrm{bp}$

$107 \rightarrow 116 \rightarrow 134 \mathrm{bp}$

124 bp

$110 \mathrm{bp}$

$126 \rightarrow 110 \mathrm{bp}$

$110 \rightarrow 127 \mathrm{bp}$

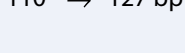

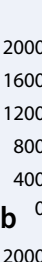$$
\text { - } 2000
$$

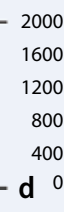

Abb. 1 Zeitlicher Verlauf. Unterschiedliche Farben in den jeweiligen Feldern kennzeichnen eine Änderung der klonalen Verwandtschaft. Positivität für LMP1 als Zeichen einer EBV-Assoziation ist durch die kleinen eingesetzten Dreiecke dargestellt (NS HL vom Subtyp der nodulären Sklerose, MC HL vom gemischtzellulären Subtyp, $L D$ lymphozytenarmer Subtyp des klassischen $\mathrm{HL}$ )

Abb. 24 Kapillarelektrophorese. Bei Patient Nr. 6 (a, c) zeigt sich ein klonaler Peak bei 94 bp in der PCR des Primärtumors (a) und ein Peak bei 95 bp - entsprechend einem klonal verwandten Rezidiv - in der Zweitneoplasie 7 Jahre später (c). Bei Patient Nr. 7 (b, d) zeigen sich Peaks von 107 bp bei dem erstdiagnostizierten $\mathrm{HL}$ (b) bzw. Peaks von 134 bp bei dem zweiten HL nach 7 Jahren (d) entsprechend einer nichtklonalen Zweitneoplasie 
globulin-Gen- (IgH-) Fragmente wurde mittels DNA-Amplifikation untersucht, wobei Consensus-FR3- und J-Primer (ABI 310 Genetic Analyzer, Applied Biosystems, USA) verwendet wurden.

\section{Ergebnisse}

Zwei frühe Erstrezidive waren mit dem ursprünglichen Lymphom klonal verwandt, 3 von 4 frühen Zweit- und Drittrezidiven waren klonal nicht miteinander verwandt (- Abb. 1, 2 a-d) Bei einem Fall kam es zusätzlich zu einer neuen Positivität für das Epstein-Barr-Virus (EBV). Die Spätrezidive von 3 Patienten waren nicht mit dem ursprünglichen Lymphom verwandt; auch hier kam es bei einem Fall zu einer neu aufgetretenen Positivität für EBV im Spätrezidiv.

\section{Schlussfolgerung}

Dies ist die bisher größte Studie, die sich mit der Frage der Klonalität von rekurrierenden $\mathrm{HL}$ durch Untersuchung von mittels Laser mikrodissezierten Hodgkinund Reed-Sternberg-Zellen auf der genetischen Ebene beschäftigt. Einige, aber nicht alle „Rezidive" sind mit dem ursprünglichen Lymphom verwandt. Diese Ergebnisse sind interessant in Bezug auf die Theorien bezüglich des Auftretens von rezidivierenden $\mathrm{HL}$ und könnten auch Auswirkungen auf deren Therapie haben.

\section{Korrespondenzadresse}

\section{PD Dr. E.C. Obermann}

Institut für Pathologie, Universitätsspital Basel Schönbeinstr. 40, 4031 Basel

Schweiz

EObermann@uhbs.ch

Interessenkonflikt. Der korrespondierende Autor gibt an, dass kein Interessenkonflikt besteht.

Pathologe 2010 · [Suppl 2] 31:132-133 DOI 10.1007/s00292-010-1343-9

(c) Springer-Verlag 2010

\section{E.C. Obermann · N. Müller · A. Rufle · T. Menter · S. Dirnhofer · A. Tzankov Klonale Verwandtschaft von Hodgkin-Lymphomen und deren Rezidiven}

\section{Zusammenfassung}

In dieser Studie untersuchten wir, ob es sich bei Rezidiven von klassischen Hodgkin-Lymphomen ( $\mathrm{HL}$ ) um Rezidive im engeren Sinn oder aber um klonal unverwandte Sekundärtumoren handelt. Die Untersuchungen erfolgten an formalinfixierten, paraffineingebetteten Gewebeproben von 11 Patienten. Hodgkin- bzw. Sternberg-Reed-Riesenzellen wurden nach immunhistochemischer Markierung mit CD30 mittels Laser mikrodisseziert und die Fragmentlängen des Schwerkettenimmunglobulin-Gens $(I g H)$ unter Verwendung von FR3- und J-Konsensusprimern analysiert. Zwei Frührezidive nach einer HL-Erstdiagnose zeigten klonale Verwandschaft zu den Primärtumoren, während 3 von 4 Frührezidiven nach einem Erst- oder Zweitrezidiv nicht mit dem vorangegangenen HL verwandt waren. Drei Spätrezidive waren mit dem ursprünglichen $\mathrm{HL}$ klonal unverwandt. Wir schließen daraus, dass es sich bei so genannten „Rezidiven“ von HL z. T. um klonal unverwandte Zweitneoplasien handeln kann, was möglicherweise von therapeutischer Relevanz sein könnte.

\section{Schlüsselwörter}

Klassisches Hodgkin-Lymphom · Klonale Verwandtschaft · Rezidiv - Lasermikrodissektion · Schwerkettenimmunglobulin-Gen (lgH)

\section{Clonal relationship of Hodgkin lymphoma and its recurrence}

\section{Abstract}

In this study, we investigated whether recurrences of classical Hodgkin's lymphoma (HL) are true relapses arising from the primary tumour or clonally unrelated secondary neoplasias. Formalin-fixed, paraffin-embedded tissue specimens of eleven patients with recurrent $\mathrm{HL}$ were analyzed. Hodgkin and ReedSternberg cells were microdissected after immunohistochemical staining for CD30 using laser-capture technique. Immunoglobulin heavy chain $(\operatorname{lgH})$ gene fragment lengths were analyzed applying consensus FR3 and J primers. Two early relapses after the first $\mathrm{HL}$ diagnosis were clonally related to the initial tumour, while three of four early recurrences after a first or second relapse were not. Three patients presenting with late relapses had clonally unrelated neoplasms. Therefore, we conclude that recurrent $\mathrm{HL}$ may represent a novel neoplasm, a finding which might play a role in clinical decision-making.

\section{Keywords}

Hodgkin lymphoma . Clonal relationship . Recurrence · Laser-microdissection · Immunoglobulin heavy chain genes 